\title{
Orbital dependent exchange-only methods for periodic systems
}

\author{
P. Süle* \\ Department Natuurkunde, University of Antwerp (RUCA), Groenenborgerlaan 171, B-2020 Antwerpen, Belgium \\ S. Kurth \\ Department of Physics and Quantum Theory Group, Tulane University, New Orleans, Louisiana 70118 \\ V. E. Van Doren \\ Department Natuurkunde, University of Antwerp (RUCA), Groenenborgelaan 171, B-2020 Antwerpen, Belgium
}

(Received 12 October 1998; revised manuscript received 17 March 1999)

\begin{abstract}
Various orbital-dependent exchange-only potentials are studied which exhibit correct long-range asymptotic behavior. We present the application of these potentials for polymers and by one of these potentials for molecules. Kohn-Sham type periodic calculations have been carried out for polyethylene in order to make valuable comparison of these potentials with each other as well as with Hartree-Fock and exchange-only LDA $(X \alpha)$ methods. The difference between total energies and highest occupied orbital energies obtained with Hartree-Fock methods and with localized exchange potentials is larger for this polymer than for atoms or molecules. Various properties of the band structure are also calculated. The band gap strongly depends on the basis set. The larger basis set makes the Kohn-Sham eigenvalue gap too low at about $4.4 \mathrm{eV}$ while the minimal basis set results in value close to the experimental gap $(\sim 8.8 \mathrm{eV})$. For the total energy and the exchange energy, the various orbital-dependent exchange-only and Hartree-Fock results differ only slightly, but for the highest occupied orbital energy the difference is more pronounced. The Kohn-Sham band gap obtained with the optimized effective potential method is corrected with the exchange contribution to the derivative discontinuity of the exchange-correlation potential. The corrected band gap obtained with the Slater's exchange potential is $9.7 \mathrm{eV}$. [S0163-1829(99)07731-0]
\end{abstract}

\section{INTRODUCTION}

Density functional theory (DFT) is well established in theoretical physics and quantum chemistry. ${ }^{1}$ The application of the theory to investigate the properties of materials has been made in the framework of the so-called second generation density functionals in which the kinetic energy is expressed in terms of orbitals but the exchange and correlation energy $E_{x c}$ in terms of the electron density. This leads to the one-electron Kohn-Sham (KS) equations. In many cases $E_{x c}$ is treated in the local density approximation (LDA). However, for atoms and molecules gradient correction to the electron density, such as the generalized gradient approximation (GGA) is necessary in order to improve the results.

Density functionals of the third generation treat both the kinetic and exchange energy exactly in terms of singleparticle orbitals and only the correlation energy needs to be approximated, either in terms of the density or in terms of the orbitals. The corresponding one-particle equations are known as the optimized effective potential method (OEP) derived some time ago by Sharp and Horton and later by Talman and Shadwick.,3 These OEP integro-differential equations yield orbitals which are implicit functionals of the density $\rho$ since the orbitals come from a local potential. Therefore, one can use the following notation for such a class of potentials: $\mathbf{v}_{x}\left[\left\{u_{i}([\rho], \mathbf{r})\right\}\right]$. The Hohenberg-Kohn theorem, ${ }^{4}$ applied to noninteracting systems, ensures that the ground-state determinant, hence, all the occupied orbitals are unique functionals of the density.

However, the OEP method is still rather complicated. Krieger, Li, and Iafrate (KLI) transformed and approximated these equations into a manageable form and applied them to atoms $^{5,6}$ where they yield results nearly identical to OEP. ${ }^{5}$ Later the KLI approach was also used for molecules ${ }^{7}$ and for certain semiconductors, ${ }^{8,9}$ but not for polymer chains. Other calculations with a Kohn-Sham potential which is believed to be close to the exact $\mathrm{KS}$ potential resulted in much narrower gap than the experiment. ${ }^{10}$

The KLI approximation to OEP provides a selfinteraction free exchange potential and proper $-1 / r$ asymptotic behavior as $r \rightarrow \infty .{ }^{5}$ Other approximate potentials $\mathrm{v}_{x}\left[\left\{u_{i}\right\}\right]$ are reported which exhibit proper asymptotics as well. ${ }^{11}$ The Koopmans theorem ${ }^{5}$ is satisfied by the highest occupied orbital in OEP and KLI, while it is violated by other density functionals such as LDA and GGA. For instance, while the popular GGA exchange-correlation energy functional $^{12}$ provides large improvements in the relative energies of various systems, the exchange-correlation potentials and the energy eigenvalues of the highest occupied state, are both suffering from significant error. ${ }^{5}$ Also others found insignificant improvement to semiconductor energy gap by GGA. ${ }^{13}$ In the last decades quite a number of publications appeared on polymer electronic structure using mainly Hartree-Fock $a b$ initio methods. ${ }^{14}$ Results are reported only the most recently, however, using DFT methods like LDA. ${ }^{15}$

In this paper we will present results obtained by various orbital dependent local exchange potentials for polymers. We give the valuable comparison of different $\mathrm{v}_{x}$ potentials which allows one to draw definite conclusions about the future applicability of OEP based methods for periodic systems. The work presented here describes the calculated band 
gaps, band widths, band structures and total energy of polyethylene within the Kohn-Sham density functional scheme using, however, various orbital dependent exchange potentials as the Slater's potential and the reasonably simplified version of the KLI exchange-only potential. For comparison we also give results obtained by Hartree-Fock (HF) and exchange-only LDA $(X \alpha)$.

\section{BASIC FORMALISM}

We start from the following Kohn-Sham effective singleparticle equation of ordinary density functional theory (for sake of simplicity we dropped the spin index and atomic units are used throughout the paper):

$$
\left[-\frac{1}{2} \nabla^{2}+\mathrm{v}_{s}(\mathbf{r})\right] u_{i}(\mathbf{r})=\epsilon_{i} u_{i}(\mathbf{r}),
$$

where $\left\{u_{i}, i=1,2, \ldots, N\right\}$ are eigenfunctions of the single particle effective $\mathrm{KS}$ potential $\mathrm{v}_{s}(\mathbf{r}) .^{1} i$ is a collective index for all single particle quantum numbers,

$$
\mathrm{v}_{s}(\mathbf{r})=\mathrm{v}_{\mathrm{ext}}(\mathbf{r})+\mathrm{v}_{H}(\mathbf{r})+\mathrm{v}_{x c}(\mathbf{r}) .
$$

As usual, $\mathbf{v}_{\text {ext }}(\mathbf{r})$ denotes the external and $\mathbf{v}_{H}(\mathbf{r})$ the Hartree potential. $\mathbf{V}_{x c}(\mathbf{r})$ is a local exchange-correlation potential which is formally defined as the functional derivative of the exchange-correlation energy

$$
\mathrm{v}_{x c}(\mathbf{r},[\rho])=\frac{\delta E_{x c}[\rho]}{\delta \rho(\mathbf{r})} .
$$

Due to the Hohenberg-Kohn theorem, there exists a one-toone mapping between the single particle $\mathrm{KS}$ potentials $\mathrm{V}_{s}(\mathbf{r})$ and densities $\rho(\mathbf{r})$ which guarantees that the functional derivative given by Eq. (3) is defined. ${ }^{1}$

The exact $E_{x c}[\rho]$ can be written as

$$
E_{x c}[\rho]=\frac{1}{2} \int d \mathbf{r}^{\prime} d \mathbf{r} \int_{0}^{1} d \lambda \frac{\rho(\mathbf{r}) \rho\left(\mathbf{r}^{\prime}\right)\left[g^{\lambda}\left([\rho] ; \mathbf{r}, \mathbf{r}^{\prime}\right)-1\right]}{\left|\mathbf{r}-\mathbf{r}^{\prime}\right|},
$$

where $g^{\lambda}\left([\rho] ; \mathbf{r}, \mathbf{r}^{\prime}\right)$ is the pair correlation function of the ficticious system with interaction strength parameter $0 \leqslant \lambda$ $\leqslant 1$ and a ground state density which is independent of $\lambda$. When employing Eq. (3) on $E_{x c}[\rho]$,

$$
\begin{aligned}
\mathbf{V}_{x c}(\mathbf{r},[\rho])= & \int_{0}^{1} d \lambda\left\{\int d \mathbf{r}^{\prime} \frac{\rho\left(\mathbf{r}^{\prime}\right)\left[g^{\lambda}\left([\rho] ; \mathbf{r}, \mathbf{r}^{\prime}\right)-1\right]}{\left|\mathbf{r}-\mathbf{r}^{\prime}\right|}\right. \\
& \left.+\frac{1}{2} \int d \mathbf{r}^{\prime} d \mathbf{r}^{\prime \prime} \frac{\rho\left(\mathbf{r}^{\prime}\right) \rho\left(\mathbf{r}^{\prime \prime}\right)}{\left|\mathbf{r}^{\prime}-\mathbf{r}^{\prime \prime}\right|} \frac{\delta g^{\lambda}\left([\rho] ; \mathbf{r}^{\prime}, \mathbf{r}^{\prime \prime}\right)}{\delta \rho(\mathbf{r})}\right\} .
\end{aligned}
$$

The first term of the potential is the so-called potential part of the exchange-correlation energy ${ }^{16,11}$ which is identical with Slater's potential in the exchange-only case, i.e., for $g^{\lambda}$ approximated by $g^{\lambda=0}$ and which is exactly known. ${ }^{17}$ To get the second, response-like term on the right-hand side of Eq. (5) one faces the technical difficulty that-even for the exchange-only case-the functional derivative cannot be determined directly. One way to get $\mathbf{v}_{x c}(\mathbf{r})$ for a given, orbitaldependent approximation to $E_{x c}$ is provided by the optimized effective potential method (OEP) given by Talman and Shadwick. ${ }^{3}$ The starting point of the OEP method is the total energy functional

$$
\begin{aligned}
E_{\mathrm{tot}}^{\mathrm{OEP}}[\rho]= & \sum_{i=1}^{\mathrm{occ}} \int d \mathbf{r} u_{i}^{*}(\mathbf{r})\left(-\frac{1}{2} \nabla^{2}\right) u_{i}(\mathbf{r}) \\
& +\int d \mathbf{r} \rho(\mathbf{r}) \mathrm{v}_{\mathrm{ext}}(\mathbf{r})+\frac{1}{2} \int d \mathbf{r} d \mathbf{r}^{\prime} \frac{\rho(\mathbf{r}) \rho\left(\mathbf{r}^{\prime}\right)}{\left|\mathbf{r}-\mathbf{r}^{\prime}\right|} \\
& +E_{x c}^{\mathrm{OEP}}\left[\left\{u_{i}\right\}\right] .
\end{aligned}
$$

In contrast to ordinary DFT, the exchange-correlation energy is an explicit functional of orbitals and therefore only an implicit functional of the density via Eq. (2). ${ }^{5,7}$ The local single-particle potential appearing in Eqs. (1) and (2) is obtained by minimizing $E_{\text {tot }}^{\mathrm{OEP}}\left[\left\{u_{i}\right\}\right]$, i.e.,

$$
\left.\frac{\delta E_{\text {tot }}^{\mathrm{OEP}}\left[\left\{u_{i}\right\}\right]}{\delta \mathrm{v}_{s}(\mathbf{r})}\right|_{\mathrm{v}_{s}=\mathrm{v}^{\mathrm{OEP}}}=0 \text {. }
$$

As pointed out by Perdew and co-workers Eq. (7) is equivalent to the Hohenberg-Kohn variational principle. ${ }^{18-20}$

The exact exchange part of $E_{x c}$ is known in terms of the single particle orbitals, i.e.,

$$
E_{x}^{\text {exact }}\left[\left\{u_{i}\right\}\right]=-\frac{1}{4} \sum_{i j}^{\text {occ }} \int d \mathbf{r} d \mathbf{r}^{\prime} \frac{u_{i}^{*}(\mathbf{r}) u_{j}^{*}\left(\mathbf{r}^{\prime}\right) u_{i}\left(\mathbf{r}^{\prime}\right) u_{j}(\mathbf{r})}{\left|\mathbf{r}-\mathbf{r}^{\prime}\right|}
$$

We will not repeat the details of the OEP calculations here and refer the interested reader to a recent review of the method. ${ }^{20}$ Also from now on we will restrict ourselves to the exchange-only case, i.e., we neglect correlation completely and use Eq. (8) as approximation to the exchange-correlation functional. The resulting OEP integral equation is rather complicated to solve in practice. ${ }^{5}$ In the work of Krieger and co-workers the OEP integral equation is analyzed and a simple approximation is made which reduces the complexity of the original OEP equation significantly and at the same time keeps many of the essential properties of OEP unchanged. ${ }^{5}$ Krieger, Li, and Iafrate gave an exact expression by transforming the OEP integral equations into a manageable form. They got the following, still exact, expression for $\mathrm{v}_{x}(\mathbf{r}):^{5}$

$$
\begin{aligned}
\mathrm{v}_{x}^{\mathrm{OEP}}(\mathbf{r})= & \mathrm{v}_{x}^{S}(\mathbf{r})+\sum_{i}^{\mathrm{occ}} \frac{\rho_{i}(\mathbf{r})}{\rho(\mathbf{r})}\left(\overline{\mathbf{v}}_{x i}^{\mathrm{OEP}}-\overline{\mathbf{v}}_{x i}^{\mathrm{HF}}\right) \\
& +\frac{1}{2} \sum_{i}^{\mathrm{occ}} \frac{\nabla\left[p_{i}(\mathbf{r}) \nabla u_{i}(\mathbf{r})\right]}{\rho(\mathbf{r})},
\end{aligned}
$$

where $\mathbf{v}_{x}^{S}(\mathbf{r})$ is the Slater potential given as the first term in Eq. (5) when $g^{\lambda}=g^{\lambda=0}$ and $\rho_{i}(\mathbf{r})=\left|u_{i}(\mathbf{r})\right|^{2}$. Slater's potential can be written in terms of the first-order density matrix $\gamma\left(\mathbf{r}, \mathbf{r}^{\prime}\right)$ as follows:

$$
\mathbf{v}_{x}^{\text {Slater }}(\mathbf{r})=-\frac{1}{2} \int \frac{\left|\gamma\left(\mathbf{r}, \mathbf{r}^{\prime}\right)\right|^{2}}{\rho(\mathbf{r})\left|\mathbf{r}-\mathbf{r}^{\prime}\right|} d \mathbf{r}^{\prime} .
$$

In Eq. (9) the summation runs over the orbital index for all the occupied orbitals up to the highest occupied $m$ th orbital (Fermi level). The function $p_{i}$ is defined by 


$$
p_{i}(\mathbf{r})=\frac{1}{u_{i}(\mathbf{r})} \int d \mathbf{r}^{\prime}\left[\mathrm{V}_{x c}^{\mathrm{OEP}}\left(\mathbf{r}^{\prime}\right)-\mathrm{V}_{i}\left(\mathbf{r}^{\prime}\right)\right] G_{i}\left(\mathbf{r}, \mathbf{r}^{\prime}\right) u_{i}\left(\mathbf{r}^{\prime}\right),
$$

where $G_{i}\left(\mathbf{r}, \mathbf{r}^{\prime}\right)$ is the Green's function

$$
G_{i}\left(\mathbf{r}, \mathbf{r}^{\prime}\right)=\sum_{j \neq i} \frac{u_{j}^{*}(\mathbf{r}) u_{j}\left(\mathbf{r}^{\prime}\right)}{\epsilon_{j}-\epsilon_{i}} .
$$

In practical applications the last term in Eq. (9) turned out to be quite small in atomic systems and has small effect only at the atomic shell boundaries. ${ }^{5}$ Krieger, Li, and Iafrate ${ }^{5}$ (KLI) have proposed a simple approximation where this last term is neglected completely. This might appear a rather crude approximation but it can be interpreted as a mean-field approximation since the neglected terms averaged over the groundstate density $\rho(\mathbf{r})$ vanish. This is not only true for finite systems with exponentially decaying densities, ${ }^{5}$ but also for infinite systems such as solids or polymers. ${ }^{20}$ The KLIapproximation to the exchange potential after some algebra reads

$$
\begin{aligned}
\mathbf{v}_{x}^{\mathrm{KLI}}(\mathbf{r})= & \mathbf{v}_{x}^{S}(\mathbf{r})+\sum_{i=1}^{m-1} \frac{\left|u_{i}(\mathbf{r})\right|^{2}}{\rho(\mathbf{r})} \sum_{j=1}^{m-1}\left(\mathbf{A}^{-1}\right)_{i j}\left(\overline{\mathbf{v}}_{x j}^{S}-\overline{\mathbf{v}}_{x j}^{\mathrm{HF}}\right) \\
& \sum_{j=1}^{m-1}\left(\mathbf{A}^{-1}\right)_{i j}\left(\overline{\mathbf{v}}_{x j}^{S}-\overline{\mathbf{v}}_{x j}^{\mathrm{HF}}\right)=\overline{\mathbf{v}}_{x i}^{\mathrm{KLI}}-\overline{\mathbf{v}}_{x i}^{\mathrm{HF}}
\end{aligned}
$$

where $m$ is the highest occupied orbital level,

$$
\begin{gathered}
\mathbf{A}_{j i}=\delta_{j i}-\mathbf{M}_{j i}, \\
\mathbf{M}_{j i}=\int \frac{\rho_{j}(\mathbf{r}) \rho_{i}(\mathbf{r})}{\rho(\mathbf{r})} d \mathbf{r}, i, j=1, \ldots, m-1 .
\end{gathered}
$$

$\overline{\mathbf{v}}_{x j}^{S}$ and $\overline{\mathbf{v}}_{x j}^{\mathrm{HF}}$ are given as follows:

$$
\begin{gathered}
\overline{\mathbf{v}}_{x j}^{S}=\int \rho_{j}(\mathbf{r}) \mathbf{v}_{x}^{S}(\mathbf{r}) d \mathbf{r}, \\
\overline{\mathbf{v}}_{x j}^{\mathrm{HF}}=-\frac{1}{2} \sum_{i}^{\mathrm{occ}} \int d \mathbf{r} d \mathbf{r}^{\prime} \frac{u_{i}^{*}(\mathbf{r}) u_{j}^{*}\left(\mathbf{r}^{\prime}\right) u_{i}\left(\mathbf{r}^{\prime}\right) u_{j}(\mathbf{r})}{\left|\mathbf{r}-\mathbf{r}^{\prime}\right|}, \\
\mathbf{v}_{x}^{S}(\mathbf{r})=-\frac{1}{2 \rho(\mathbf{r})} \sum_{i, j}^{\mathrm{occ}} u_{i}^{*}(\mathbf{r}) u_{j}(\mathbf{r}) \int d \mathbf{r}^{\prime} \frac{u_{i}\left(\mathbf{r}^{\prime}\right) u_{j}^{*}\left(\mathbf{r}^{\prime}\right)}{\left|\mathbf{r}-\mathbf{r}^{\prime}\right|}, \\
\mathbf{v}_{i}(\mathbf{r})=\frac{1}{u_{i}^{*}(\mathbf{r})} \frac{\delta E_{x}^{\mathrm{OEP}}\left[\left\{u_{i}\right\}\right]}{\delta u_{i}(\mathbf{r})} .
\end{gathered}
$$

The term corresponding to the highest occupied orbital $u_{m}$ has been excluded from the sum in Eq. (13), because $\overline{\mathbf{V}}_{x m}^{\mathrm{KLI}}$ $=\overline{\mathrm{v}}_{x m}^{\mathrm{HF}} 5$

In this article we restrict ourselves to different exchangeonly methods, however, the extension of these methods to exchange-correlation case is in principle straightforward. ${ }^{5}$ The most trivial way of accounting for Coulomb correlation effects is the use of a correlation energy functional either in its local or gradient corrected form. However, recent calcu- lations using a gradient- and orbital-dependent functional in combination with exact exchange provided excellent results for atoms only. ${ }^{7}$ The poor performance of this functional for molecules must be attributed to the improper long-range component in the corresponding correlation hole which is needed to cancel the long-range component in the exact exchange hole (the combined $x c$ hole is typically short-ranged). Most of the approximate correlation functionals, which are derived from the homogeneous or the inhomogeneous electron gas model, are suffering from incorrect long-range assymptotics and exhibit improper local behavior. ${ }^{16}$ Therefore the extension of OEP based methods to include electron correlation will probably be the subject of further studies in the near future. ${ }^{21}$

In this paper we do not attempt to solve Eq. (13) for polymers but instead choose a different approach proposed by Gritsenko et al. ${ }^{11}$ For the constants $w_{j}=\overline{\mathrm{V}}_{x j}^{\mathrm{KLI}}-\overline{\mathrm{V}}_{x j}^{\mathrm{HF}}$ in Eq. (13) an alternative expression is proposed ${ }^{11}$ in terms of orbital energies $\epsilon_{j}$. It follows from gauge invariance requirements, proper scaling and short range behavior of the response part of Eq. (5) and Eq. (13) that $w_{j}$ does only depend on energy differences. Therefore Eq. (13) turns into the following much simpler formula:

$$
\mathrm{v}_{x}^{\mathrm{SSP}}(\mathbf{r})=\mathrm{v}_{x}^{S}(\mathbf{r})+\frac{8 \sqrt{2}}{3 \pi^{2}} \sum_{i=1}^{m-1} \frac{\left|u_{i}(\mathbf{r})\right|^{2}}{\rho(\mathbf{r})} \sqrt{\epsilon_{F}-\epsilon_{i}}
$$

where $\epsilon_{F}$ is the Fermi level (highest occupied energy level). We use the notation SSP (Slater's potential + step potential) for this exchange potential. The constant $8 \sqrt{2} / 3 \pi^{2}$ is obtained from the homogeneous electron gas (HEG) model so that Eq. (20) is exact in the HEG limit. ${ }^{11}$ This constant is chosen as universal parameter for all the calculations. It is one of the main advantages of this expression that one can avoid the matrix inversion of Eq. (13) and also suggests the future applicability of a more general class of $\mathrm{v}_{x c}$ which can be designated by $\mathrm{v}_{x c}\left(\left[\left\{u_{i}\right\},\left\{\boldsymbol{\epsilon}_{i}\right\}\right] ; \mathbf{r}\right) .{ }^{21}$ Note that the summation runs over all the occupied orbitals except the highest one, as in Eq. (13). With the step potential-like second term $\mathrm{v}_{x}^{\mathrm{SSP}}$ provides a good approximation to the OEP exchangepotential [Eq. (9)] (Ref. 11) that possesses proper short-range behavior and the characteristic atomic-shell stepped structure.

\section{THE IMPLEMENTATION OF THE ORBITAL DEPENDENT EXCHANGE POTENTIALS}

In this section we briefly give the summary of the theory for a periodic one-dimensional polymer chain based on Gaussian lobe functions. ${ }^{22}$

In the following we want to describe quasi one dimensional helical chain polymers, i.e., we consider a single polymer chain (extending to infinity) which may or may not have a helix structure (for general background on the LCAO method on helical polymers see, e.g., Ref. 15). Mathematically this symmetry can be expressed by a screw operation $\mathbf{S}$ in terms of a translation $a$ along the $z$-axis combined with a rotation of angle $\Theta$ about the same axis, i.e., formally, 


$$
\mathbf{S}(a, \Theta) \mathbf{r}=\left(\begin{array}{c}
x \cos \Theta-y \sin \Theta \\
x \sin \Theta+y \cos \Theta \\
z+a
\end{array}\right) \text {. }
$$

The symmetry group generated by the screw operation is an Abelian group and therefore its irreducible representations are one-dimensional. Therefore the single-electron wave functions corresponding to this problem will transform according to

$$
u_{i}\left(k, \mathbf{S}^{n} \mathbf{r}\right)=\exp (-i k n) u_{i}(k, \mathbf{r}),
$$

where the label $k$ (which may be viewed as a normalized wave number) is restricted to the range $-\pi \leqslant k \leqslant \pi$ and $i$ is simply the band index (to avoid confusion of indexes we note that $i$ in the exponent is simply the imaginary number).

The electrons are assumed to doubly occupy a set of oneelectron orbitals, $u_{i}(k, \mathbf{r})$, of Bloch-type. These orbitals are written as linear combinations of $m_{b}$ atomic (real) basis functions. $\left\{\chi_{\mu}, \mu=1, \ldots, m_{b}\right\}$ denotes the basis atomic func- tions in the reference unit cell and $\chi_{\mu}^{n}(\mathbf{r})=\chi_{\mu}^{0}\left(\mathbf{S}^{n} \mathbf{r}\right)$ are the corresponding basis functions for unit cell $n$. The ansatz for the orbitals then reads

$$
u_{i}(k, \mathbf{r})=\sum_{n=-\infty}^{+\infty} \sum_{\mu}^{m_{b}} c_{i \mu}(k) e^{i k n} \chi_{\mu}^{n}(\mathbf{r})
$$

which transforms according to Eq. (22). In this article the sets of index $\left(n, n^{\prime}, n^{\prime \prime}\right),(i, j)$ and $(\mu, \nu, \sigma, \tau)$ are used, referring to cells, orbitals and contracted atomic lobe functions. The unknown coefficients $c_{i \mu}(k)$ have to be determined through the self-consistency procedure. Since we are dealing with an infinite chain polymer, the sum over the neighboring cells extends from $-\infty$ to $+\infty$. In practice, however, we only take a finite number of neighboring cells into account. In Sec. V we will address the question of how many cells need to be taken into account in order to get converged groundstate energies.

The total energy for the polymer system is expressed as ${ }^{15}$

$$
\begin{aligned}
E_{\mathrm{tot}}= & \sum_{\mu \nu}^{m_{b}} \sum_{n=-\infty}^{+\infty} \mathbf{P}_{\mu \nu}^{0, n}\left\{-\frac{1}{2}\left\langle\chi_{\mu}^{0}\left|\nabla^{2}\right| \chi_{\nu}^{n}\right\rangle+\left\langle\chi_{\mu}^{0}\left|e_{x c}\left(\left[\left\{u_{i}\right\}\right] ; \mathbf{r}\right)\right| \chi_{\nu}^{n}\right\rangle\right\}+\frac{1}{2} \sum_{n^{\prime}=-\infty}^{+\infty}\left\{\left(\sum_{r s} \frac{Z_{r} Z_{s}}{\left.\left|\mathbf{R}_{r}^{0}-\mathbf{R}_{s}^{n^{\prime}}\right|\right)}\right.\right. \\
& \left.+\sum_{\mu \nu}^{m_{b}} \sum_{n=-\infty}^{+\infty} \mathbf{P}_{\mu \nu}^{0, n}\left(\sum_{\sigma \tau}^{m_{b}} \sum_{n^{\prime \prime}=-\infty}^{+\infty} \mathbf{P}_{\sigma \tau}^{n^{\prime}, n^{\prime \prime}}\left\langle\chi_{\mu}^{0} \chi_{\nu}^{n} \mid \chi_{\sigma}^{n^{\prime}} \chi_{\tau}^{n^{\prime \prime}}\right\rangle-2 \sum_{r}\left\langle\chi_{\mu}^{0}\left|\frac{Z_{r}}{\left|\mathbf{r}-\mathbf{R}_{r}^{n^{\prime \prime}}\right|}\right| \chi_{\nu}^{n}\right\rangle\right)\right\},
\end{aligned}
$$

where $Z_{r}$ and $\mathbf{R}_{r}$ denote the nuclear charges and coordinates within a single unit cell, $\mathbf{R}_{r}^{n}$ denotes the nuclear coordinates in unit cell $n\left(\mathbf{R}_{r}^{n}=S^{n} \mathbf{R}_{r}\right)$. In our particular case the orbital dependent exchange-correlation energy density is $e_{x c}\left(\left[\left\{u_{i}\right\}\right] ; \mathbf{r}\right)=e_{x}\left(\left[\left\{u_{i}\right\}\right] ; \mathbf{r}\right) \quad$ and also $E_{x}=\int e_{x}\left(\left[\left\{u_{i}\right\}\right] ; \mathbf{r}\right) d \mathbf{r}$. $^{16}$

According to Eq. (8) $E_{x}^{\mathrm{OEP}}$ can be given in terms of oneparticle density matrixes as well,

$$
\begin{aligned}
E_{x}^{\mathrm{OEP}}= & -\frac{1}{4} \sum_{\mu \nu} \sum_{n, n^{\prime}, n^{\prime \prime}=-\infty}^{+\infty} \mathbf{P}_{\mu \nu}^{0, n} \sum_{\sigma \tau} \mathbf{P}_{\sigma \tau}^{n^{\prime}, n^{\prime \prime}} \\
& \times \int \frac{\chi_{\mu}^{0}(\mathbf{r}) \chi_{\nu}^{n}\left(\mathbf{r}^{\prime}\right) \chi_{\sigma}^{n^{\prime}}(\mathbf{r}) \chi_{\tau}^{n^{\prime \prime}}\left(\mathbf{r}^{\prime}\right)}{\left|\mathbf{r}-\mathbf{r}^{\prime}\right|} d \mathbf{r}^{\prime} d \mathbf{r},
\end{aligned}
$$

where $\chi_{\mu}^{0}, \chi_{\nu}^{n}, \chi_{\sigma}^{n^{\prime}}$ and $\chi_{\tau}^{n^{\prime \prime}}$ are the basis functions in which the orbitals are expanded. The upper indexes 0 and $n, n^{\prime}, n^{\prime \prime}$ denote the localization of the basis function in the reference cell and in the infinite system, respectively. The density matrix elements for one-dimensional periodic systems are given by

$$
\mathbf{P}_{\sigma \tau}^{n^{\prime}, n^{\prime \prime}}=\sum_{j=1}^{\text {occ }} \int_{-\pi}^{\pi} \frac{d k}{2 \pi} c_{j \sigma}^{*}(k) c_{j \tau}(k) \exp \left[i k\left(n^{\prime}-n^{\prime \prime}\right)\right] .
$$

The density matrix $\mathbf{P}_{\mu \nu}$ is computed at each iteration by numerical integration over the occupied part of the first Bril- louin zone of the polymer. The interactions of the "reference cell", with neighbors (finite neighboring interaction) are taken into account by the summation over the cell index $n$. The electron density can then be written as

$$
\rho(\mathbf{r})=\sum_{n=-\infty}^{+\infty} \sum_{\mu \nu}^{m_{b}} \mathbf{P}_{\mu \nu}^{0, n} \chi_{\mu}^{0}(\mathbf{r}) \chi_{\nu}^{n}(\mathbf{r})
$$

The contracted Gaussian-lobe $\chi_{\mu}(\mathbf{r})$ basis functions in the reference unit cell ${ }^{22}$ is equal to

$$
\chi_{\mu}(\mathbf{r})=\frac{1}{\sqrt{N}} \sum_{\alpha} d_{\mu \alpha} \exp \left(-\alpha_{\mu \alpha}\left|\mathbf{r}-\mathbf{A}_{\mu \alpha}-\mathbf{R}_{s}\right|^{2}\right)
$$

where the summation runs over contraction index $\alpha$. The constants $d_{\mu \alpha}$ are fixed according to the contraction pattern. The basis functions $\left\{\chi_{\mu}(\mathbf{r}), \mu=1, \ldots, m_{b}\right\}$ are also chosen to be normalized and $\mathbf{R}_{s}$ is the atomic position in the unit cell from where the lobe functions are displaced. In the basis set the higher angular momentum terms $p, d, \ldots$ are treated by linear combinations of $s$-functions (lobes) with its origins displaced from the nuclear position by vectors $\mathbf{A}_{\mu \alpha}$ (for further details see Ref. 22). For instance, a $p_{x}$ orbital can be written as a difference between two lobe functions $u_{p_{x}}$ $=1 / \sqrt{N}\left(u_{1}-u_{2}\right)$ so that the exponent in the basis set will be 


$$
\left|\mathbf{r}-\mathbf{A}_{\mu \alpha}-\mathbf{R}_{\mathbf{s}}\right|^{2}=\left[r_{x}+(-1)^{l} A-R_{s x}\right]^{2}+\sum_{i=y, z}\left(r_{i}-R_{s i}\right)^{2},
$$

where $A$ is the magnitude of the displacement of the lobe centers, and $R_{s i}$ is the component of the position vector of nucleus $s$. According to Eq. (18), the Slater potential in terms of Gaussian basis sets and of the self-consistently determined density matrix can be written

$$
\begin{aligned}
\mathbf{V}_{x}^{\text {Slater }}(\mathbf{r})= & -\frac{1}{2 \rho(\mathbf{r})} \sum_{n, n^{\prime}, n^{\prime \prime}=-\infty}^{+\infty} \sum_{\mu \nu} \mathbf{P}_{\mu \nu}^{0, n} \\
& \times \sum_{\sigma \tau} \mathbf{P}_{\sigma \tau}^{n,{ }^{\prime} n^{\prime \prime}} \chi_{\mu}^{0}(\mathbf{r}) \chi_{\sigma}^{n^{\prime}}(\mathbf{r}) \int \frac{\chi_{\nu}^{n}\left(\mathbf{r}^{\prime}\right) \chi_{\tau}^{n^{\prime \prime}}\left(\mathbf{r}^{\prime}\right)}{\left|\mathbf{r}-\mathbf{r}^{\prime}\right|} d \mathbf{r}^{\prime} .
\end{aligned}
$$

While the integration with respect to the primed $\mathbf{r}^{\prime}$ coordinate can be carried out analytically, ${ }^{23}$ the second integration to calculate the matrix elements according to Eq. (16) can be accomplished only numerically which makes the procedure somewhat time consuming. However, further attention will be devoted to simplifying this integral using auxiliary fit functions. ${ }^{15}$

Since we are dealing with a Hartree-Fock-Roothan-Hall SCF-LCAO-MO approximation in a basis set representation, ${ }^{24}$ one has to apply the Fock matrix formalism and has to form the corresponding Fock matrix elements:

$$
\mathbf{F}_{\mu \nu}^{n}=\mathbf{H}_{\mu \nu}^{n}+\mathbf{J}_{\mu \nu}^{n}+\mathbf{F}_{\mu \nu}^{n, x},
$$

where $\mathbf{H}_{\mu \nu}^{n}, \mathbf{J}_{\mu \nu}^{n}$ and $\mathbf{F}_{\mu \nu}^{n, x}$ are the one-electronic, Coulomb and exchange contributions to the Fock matrix $\mathbf{F}_{\mu \nu}^{n}$ with $n$ unit cell so that

$$
\begin{gathered}
\mathbf{H}_{\mu \nu}^{n}=\left\langle\chi_{\mu}^{0}\left|-\frac{1}{2} \nabla+\mathrm{v}_{\mathrm{ext}}(\mathbf{r})\right| \chi_{\nu}^{n}\right\rangle, \\
\mathbf{J}_{\mu \nu}^{n}=\left\langle\chi_{\mu}^{0}\left|\mathbf{v}_{H}(\mathbf{r})\right| \chi_{\nu}^{n}\right\rangle,
\end{gathered}
$$

and

$$
\mathbf{F}_{\mu \nu}^{n, x}=\left\langle\chi_{\mu}^{0}\left|\mathbf{v}_{x}(\mathbf{r})\right| \chi_{\nu}^{n}\right\rangle,
$$

where $\mathbf{v}_{H}(\mathbf{r})$ is the Coulomb term of the Hartree potential. According to Eq. (1) and Eq. (23) the stationary one-electron periodic wave functions $\left\{u_{i}(k, \mathbf{r})\right\}$ are obtained in the usual way by solving the eigenvalue equations

$$
\begin{gathered}
\sum_{\mu} c_{j \mu}(k)\left\{\sum_{n=-\infty}^{+\infty} e^{i k n}\left[\mathbf{F}_{\mu \nu}^{n}-\boldsymbol{\epsilon}_{j}(k) \mathbf{S}_{\mu \nu}^{n}\right]\right\}=0, \\
\mu, \nu=1,2, \ldots, m_{b}
\end{gathered}
$$

with

$$
\mathbf{S}_{\mu \nu}^{n}=\int d \mathbf{r} \chi_{\mu}^{0}(\mathbf{r}) \chi_{\nu}^{n}(\mathbf{r}),
$$

at a finite number of $k$ points in the Brillouin zone (BZ). This gives the band structure $\epsilon_{j}(k)$ and the eigenvectors.

\section{COMPUTATIONAL DETAILS}

On the basis of the formalism described above the total energy and the electronic band structure have been calculated for polyethylene by various exchange-only methods. All of these schemes can be considered as orbital dependent methods and results are compared with other density functional exchange-only methods like the simple exchange-only LDA $(X \alpha)$ procedure. Hartree-Fock calculations have been carried out as well to make valuable comparison with the previous methods. For all the calculations a code is used which has been developed in our group and the results are compared with other polymer codes like the Mintmire's program. ${ }^{15}$ This code is based on the Erlangen periodic Hartree-Fock program ${ }^{25}$ and has been developed by Kurth and Süle. ${ }^{26}$ During the calculations the number of $k$-points and the convergence criteria are set to 25 and $10^{-5}$ (the change of the density matrix elements from one iteration to the next), respectively. For polyethylene the experimental "zig-zag" geometry is used. ${ }^{15}$ Since the various properties of polyethylene depend significantly on the geometry, we give the structure we have used for the calculations: the $\mathrm{C}-\mathrm{C}$ and $\mathrm{C}-\mathrm{H}$ bond lengths and the $\mathrm{CCC}, \mathrm{HCH}, \mathrm{HCC}$ bond angles are set to $1.54 \AA, 1.10 \AA$, and $113^{\circ}, 108^{\circ}$, and $113^{\circ}$, respectively. Recent geometry optimization calculations with various DFT functionals provided values in very good agreement with these experimental bonding parameters. ${ }^{27}$ Three types of basis sets are used: Clementi's minimal $(7 \mathrm{~S} / 3 \mathrm{P})$, the larger double $-\zeta(9 \mathrm{~S} / 5 \mathrm{P})$ and $(11 \mathrm{~S} / 7 \mathrm{P})$ basis sets for carbon, and the $4 \mathrm{~S}, 6 \mathrm{~S}$ and $8 \mathrm{~S}$ for the hydrogen atom, respectively. ${ }^{28}$ To make valuable comparison with previously published atomic and molecular results ${ }^{6,7}$ and also to check the reliability of our code we have made calculations with a translation vector of about 20.0 a.u. and with the minimal number of neighboring interactions $($ neig $=1)$. Using these parameters the computed properties must be very close to those obtained by atomic or molecular codes. The test provided nice agreement with atomic calculations obtained by Hartree-Fock, Slater's or SSP exchange-only method which confirms the reliability of our code.

\section{RESULTS AND DISCUSSION}

The comparison is carried out for $\mathrm{Be}$ and for $\mathrm{LiH}$ (Refs. 7 and 11) (Table I) in the above-mentioned atomic and molecular limit with our polymer code ${ }^{26}$ The deviation of the total energies from $\mathrm{HF}$ can be compared with results obtained by others: ${ }^{11}$ we found 12,5 mHartree for Slater and SSP, while Gritsenko et al. obtained 11 and 0.0, respectively. For LiH the deviations: $0.0,0.0 \mathrm{mH}$, while Grabo et $\mathrm{al}^{7}$ found $6 \mathrm{mH}$ for Slater. The discrepancy between our and other's results can be considered rather small and is probably due to the different basis set applied here. The total energies for $\mathrm{C}_{2} \mathrm{H}_{4}$ are compared as well in the molecular limit and the following values are obtained with Hartree-Fock, Slater and SSP exchange-only methods: $-77.61066,-77.58735$, and -77.60790 a.u. One can see that the deviation from HF is 24 $\mathrm{mH}$ for Slater and only $3 \mathrm{mH}$ for SSP. For the highest occupied orbital energies we got $-0.4273,-0.4878$, and -0.3854 a.u. by HF, Slater, and SSP. For LiH the corresponding numbers are as follows: $-0.2914,-0.3228$, and -0.3088 a.u. which are compared with Grabo's values, ${ }^{7}$ -0.3017 (HF), -0.3150 (Slater), and -0.3011 (KLI). We also compared our calculated HF total energy and Fermi level with those obtained by other $a b$ initio codes like the 
TABLE I. Comparison of HF, Slater, and SSP results for Be and LiH obtained with the DIOGENES code using Clementi's minimal basis set with values from the literature.

\begin{tabular}{|c|c|c|c|c|c|c|c|}
\hline & & $\mathrm{HF}^{\text {our }}$ & $\mathrm{HF}^{\text {other }}$ & Slater ${ }^{\text {our }}$ & Slater ${ }^{\text {other }}$ & $\mathrm{SSP}^{\text {our }}$ & $\mathrm{SSP}^{\text {other }}$ \\
\hline $\mathrm{Be}$ & $E_{\text {tot }} /$ a.u. & -14.567 & $-14.573^{\mathrm{a}}$ & -14.556 & $-14.551^{b}$ & -14.562 & $-14.560^{\mathrm{b}}$ \\
\hline \multirow[t]{2}{*}{$\mathrm{LiH}$} & $E_{\text {tot }} /$ a.u. & $\begin{array}{l}-7.954 \\
-7.8629^{\mathrm{e}}\end{array}$ & $\begin{array}{l}-7.987^{\mathrm{c}} \\
-7.8620^{\mathrm{e}}\end{array}$ & -7.954 & $-7.981^{\mathrm{c}}$ & -7.954 & $-7.987^{\mathrm{c}}$ \\
\hline & $\epsilon_{m} /$ a.u. & $\begin{array}{l}-0.2914 \\
-0.2870^{\mathrm{d}}\end{array}$ & $\begin{array}{l}-0.3017^{\mathrm{c}} \\
-0.2857^{\mathrm{e}}\end{array}$ & -0.3228 & $-0.3150^{\mathrm{c}}$ & -0.3088 & $-0.3011^{\mathrm{c}}$ \\
\hline
\end{tabular}

${ }^{\mathrm{a}}$ Reference 37.

${ }^{\mathrm{b}}$ Reference 11 .

${ }^{\mathrm{c}}$ Reference 7.

${ }^{\mathrm{d}}$ With STO-3G basis.

${ }^{\text {e}}$ With STO-3G basis using GAUSSIAN94 (Ref. 29) program package.

GAUSSIAN 94 package $^{29}$ for $\mathrm{LiH}$ using the STO-3G minimal basis set. The agreement is acceptable which also indicates that our code works in a proper way.

Our results for some physical properties of polyethylene are shown in Tables II and III. The results are listed in terms of the number of the neighboring interactions. Actually, it turns out that at least five neighbors are necessary to get converged results for all the physical properties we are interested in. For comparison we have also calculated the properties for eight neighboring cells. In Table III values are given obtained from Mintmire's polymer code ${ }^{15}$ as well, which is using the multiple expansion technique for the neighboring interaction and can therefore be considered as an infinite order approach. ${ }^{15}$ However, in this code a different basis set is used $(721 / 51 / 1 *)$ which makes the comparison somewhat more difficult.

The comparison of total energies shows that Hartree-Fock provides the lowest energies while the various Kohn-Sham schemes result in somewhat higher energies. OEP leads to total energy values that are upper bounds to the HF results and generally the inequality, $E_{\mathrm{HF}}<E_{\mathrm{OEP}}<E_{\mathrm{KLI}}<E_{\mathrm{LSDX}}$ holds. ${ }^{5}$ Actually the difference between HF and SSP is 10

TABLE II. Calculated properties of polyethylene by various DFT methods using the minimal basis set. HF, Slater, SSP, and $X \alpha$ denote the Hartree-Fock, exchange-only method with Slater's potential [see Eq. (16)], Slater's potential with orbital dependent step potential Eq. (20), and the $X \alpha$ exchange-only density functional method, respectively. All the properties are in a.u. except the vertical HOMO-LUMO eigenvalue gap which is given in $\mathrm{eV}$ and which is computed at the edge of the Brillouin zone $(k=1)$. Neig is the number of the neighbors taken into account in the neighboring approach. $E_{\text {tot }} / u, E_{x}$ are the calculated total and exchange energies per unit cell. $\epsilon_{m}$ and $\epsilon_{m+1}$ are the highest occupied and the first virtual energy levels. $\Delta_{x}$ is the exchange contribution to the total derivative discontinuity. The calculations were carried out on the $\mathrm{CH}_{2}$ unit cell.

\begin{tabular}{|c|c|c|c|c|c|}
\hline Neig & & $\mathrm{HF}$ & Slater & SSP & $X \alpha$ \\
\hline 1 & $\begin{array}{c}E_{\text {tot }} / \mathrm{u} \text { (a.u.) } \\
E_{x} \text { (a.u.) } \\
\epsilon_{m} \text { (a.u.) } \\
\epsilon_{m+1} \text { (a.u.) } \\
\text { gap (eV) } \\
\Delta_{x}(\mathrm{eV})\end{array}$ & $\begin{array}{c}-39.03535 \\
-5.82128 \\
-0.6672 \\
0.3978 \\
29.0\end{array}$ & $\begin{array}{c}-39.01888 \\
-5.84774 \\
-0.4321 \\
-0.4083 \\
15.1 \\
-5.7\end{array}$ & $\begin{array}{c}-39.02768 \\
-5.79080 \\
-0.4924 \\
-0.1084 \\
16.4 \\
-4.4\end{array}$ & $\begin{array}{c}-38.41375 \\
-5.15416 \\
-0.5070 \\
-0.1535 \\
18.0\end{array}$ \\
\hline 5 & & $\begin{array}{c}-38.88012 \\
-5.75048 \\
-0.4747 \\
0.3444 \\
22.3\end{array}$ & $\begin{array}{c}-38.86891 \\
-5.76397 \\
-0.3524 \\
-0.0433 \\
8.4 \\
-3.5\end{array}$ & $\begin{array}{c}-38.87278 \\
-5.7379 \\
-0.2312 \\
0.1310 \\
9.9 \\
-1.9\end{array}$ & $\begin{array}{c}-38.30351 \\
-5.16982 \\
-0.2761 \\
0.1514 \\
11.6\end{array}$ \\
\hline 8 & & $\begin{array}{c}-38.88008 \\
-5.75049 \\
-0.4760 \\
0.3436 \\
22.3\end{array}$ & $\begin{array}{c}-38.86890 \\
-5.76400 \\
-0.3518 \\
-0.0425 \\
8.4 \\
-3.5\end{array}$ & $\begin{array}{c}-38.87275 \\
-5.7378 \\
-0.2318 \\
0.1303 \\
9.9 \\
-1.9\end{array}$ & $\begin{array}{c}-38.30348 \\
-5.16983 \\
-0.2769 \\
0.1510 \\
11.6\end{array}$ \\
\hline
\end{tabular}


TABLE III. Calculated properties of polyethylene by various DFT methods using the Clementi's doublezeta basis set $(9 \mathrm{~S} / 5 \mathrm{P})$ and the $(11 \mathrm{~S} / 7 \mathrm{P})$ one. The notations are the same as in Table II.

\begin{tabular}{|c|c|c|c|c|c|}
\hline Neig & & $\mathrm{HF}$ & Slater & SSP & $X \alpha$ \\
\hline $5(9 \mathrm{~S} / 7 \mathrm{P})$ & $\begin{array}{c}E_{\text {tot }} / \mathrm{u} \\
E_{x} \\
\epsilon_{m} \\
\epsilon_{m+1} \\
\text { gap } \\
\Delta_{x}\end{array}$ & $\begin{array}{c}-39.01068 \\
-5.8783 \\
-0.4049 \\
0.1489 \\
15.1\end{array}$ & $\begin{array}{c}-38.96683 \\
-5.9368 \\
-0.2951 \\
-0.1590 \\
3.7 \\
3.4\end{array}$ & $\begin{array}{c}-38.99704 \\
-5.8841 \\
-0.1628 \\
0.0007 \\
4.4 \\
3.8\end{array}$ & $\begin{array}{c}-38.41919 \\
-5.2527 \\
-0.2227 \\
0.0256 \\
6.8\end{array}$ \\
\hline $8(9 \mathrm{~S} / 7 \mathrm{P})$ & & $\begin{array}{c}-39.01056 \\
-5.8780 \\
-0.3984 \\
0.1558 \\
15.1\end{array}$ & $\begin{array}{c}-38.96684 \\
-5.9367 \\
-0.2774 \\
-0.1424 \\
3.7 \\
3.2\end{array}$ & $\begin{array}{c}-38.99700 \\
-5.8832 \\
-0.1490 \\
0.0116 \\
4.4 \\
3.7\end{array}$ & $\begin{array}{c}-38.4190 \\
-5.25221 \\
-0.2116 \\
0.0375 \\
6.8\end{array}$ \\
\hline $8(11 \mathrm{~S} / 7 \mathrm{P})$ & & $\begin{array}{c}-39.02326 \\
-5.8980 \\
-0.3786 \\
0.2320 \\
16.6\end{array}$ & $\begin{array}{c}-38.99918 \\
-5.9486 \\
-0.2401 \\
-0.1151 \\
3.4 \\
6.3\end{array}$ & $\begin{array}{c}-38.01359 \\
-5.9036 \\
-0.1130 \\
0.0755 \\
5.1 \\
7.6\end{array}$ & $\begin{array}{c}-38.42701 \\
-5.2658 \\
-0.2421 \\
0.0354 \\
7.6\end{array}$ \\
\hline$\sim \infty$ & gap (Expt.) & & & & $\begin{array}{c}-38.45548 \\
-5.27507 \\
-0.2111 \\
0.0750 \\
7.4 \\
8.8^{\mathrm{a}}\end{array}$ \\
\hline
\end{tabular}

${ }^{\mathrm{a}}$ References 15 and 31.

$\mathrm{mH}$. For small diatomic molecules Grabo and Gross $^{7}$ found a difference of about $10 \mathrm{mH}$ between KLI and HF as well, which certainly indicates that the SSP potential given by Eq. (20) is not a bad alternative for KLI. For a larger basis set the SSP and HF total energies differ more significantly $(14 \mathrm{mH})$, which is, however, much smaller than the corresponding difference for Slater $(44 \mathrm{mH})$. We believe that the bulk part of the difference is not due to the approximation of the exchange potentials applied here, but to the different nature of Hartree-Fock and DFT approaches in accordance with earlier studies on molecules. ${ }^{5,7}$ However, in a polymer chain calculation we get for the one electronic energies quite significant deviation from the Hartree-Fock values (Tables II and III). There is a trend in these results: the employment of exchange potentials results in the raising of the Fermi level and the falling of the first virtual levels giving a smaller gap when compared with Hartree-Fock for polyethylene. $\epsilon_{m}^{\mathrm{HF}}$ remains almost constant with respect to the increase of the number of neighbors taken into account in the neighboring approach.

Neglect of the response part in Eq. (13) leads to somewhat higher energies for $E_{\text {tot }}$. Thus the addition of a repulsive response part to the attractive bare Slater potential brings $E_{\text {tot }}$ and $E_{x}$ closer to the corresponding Hartree-Fock values. ${ }^{11}$ The error in $E_{\text {tot }}$ obtained with the naked Slater potential $\mathrm{v}_{x}^{S}$ increases with the basis set and reaches $20 \mathrm{mH}$ for the largest basis set when compared with SSP.
Tables II and III represent exchange $E_{x}$ energies as well. Slater yields too negative $E_{x}$ because of the attractive character of $\mathbf{V}_{x}^{S}$. The addition of the approximate repulsive step potential (SSP) brings the $E_{x}$ values much closer to $E_{x}^{\mathrm{HF}}$ but still remains somewhat more negative (Table III). Gritsenko et al. ${ }^{11}$ found, however, the overcompensation of $E_{x}^{\mathrm{SSP}}$ values compared with $E_{x}^{\mathrm{OEP}}$ for atoms. The inequality $E_{x}^{\mathrm{OEP}} \leqslant E_{x}^{\mathrm{HF}}$ (Ref. 30) is clearly reproduced by Slater and SSP with the larger basis set.

The most striking feature to be mentioned is that the highest occupied orbital energies $\epsilon_{m}$ obtained by SSP differ from HF values significantly, and are less negative than $\epsilon_{m}^{X \alpha}$. These results are rather surprising since atomic and molecular calculations show that the $\epsilon_{m}$ values obtained by HF or OEP are close to each other. ${ }^{5-7}$ While this comes as a surprise we consider the raising of the Fermi level due to the periodic effects, which come into play only, however, when one employs localized exchange-potentials for infinite systems. One can see how the periodic effect comes into play when the change of $\epsilon_{m}$ is examined in terms of the order of the neighboring interactions in Table II. As may be read from Table II, when neig $=1$ (which case is closer to the molecular limit than to a periodic system), $\epsilon_{m}$ obtained by Slater or SSP is rather close to the HF value, however, when neig is increased the Fermi level shifted upward and is get- 


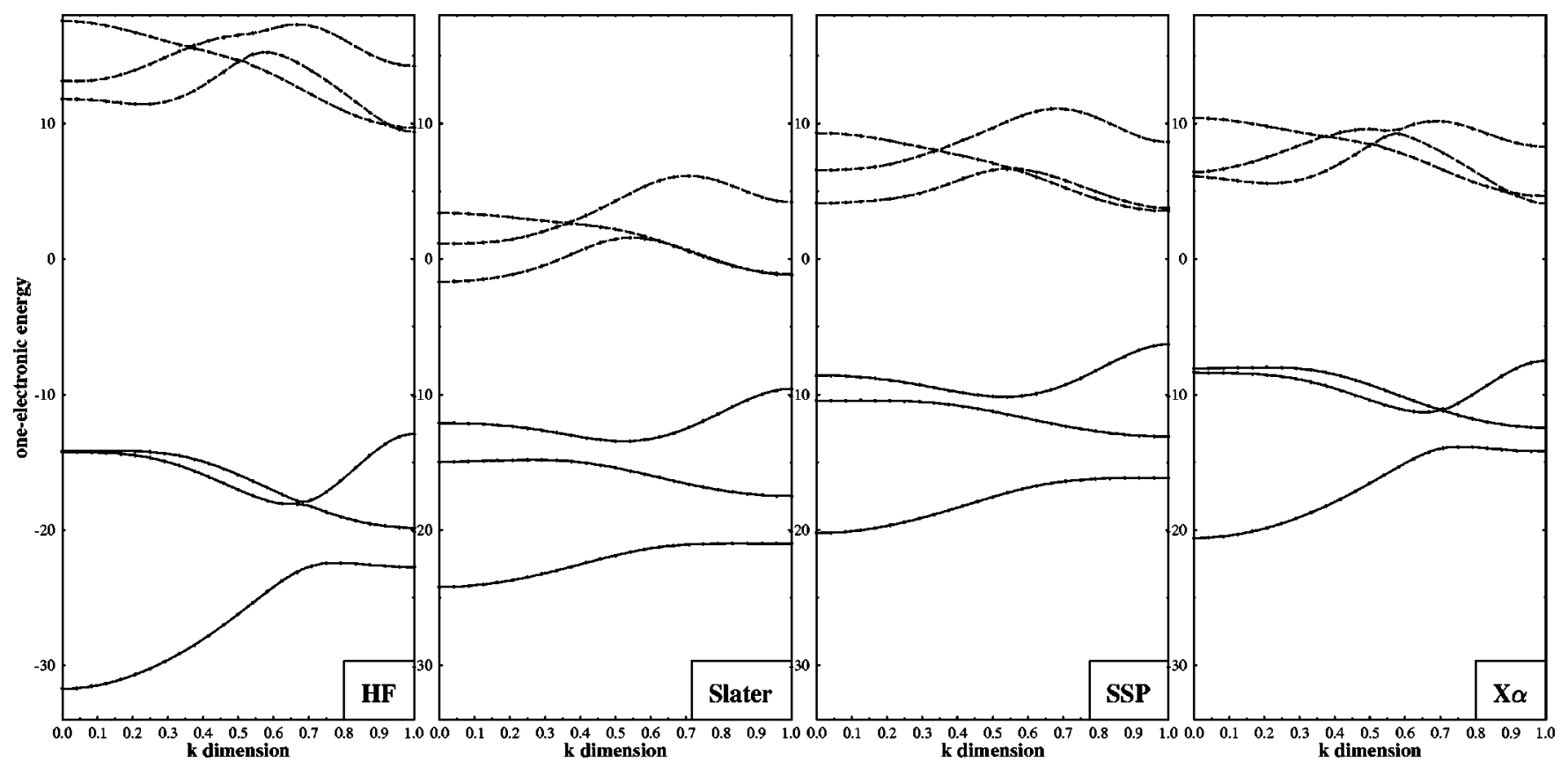

FIG. 1. The calculated valence band structure $(\mathrm{eV})$ obtained by various exchange-only methods as a function of the dimensionless $k$ variable with $k=0$ being the zone center and $k=1$ the zone edge. The Brillouin zone is that corresponding to the $\mathrm{CH}_{2}$ unit cell. Solid lines correspond to occupied and dashed lines to virtual levels. The Clementi's minimal basis set is used and five neighbors are considered for the $\mathrm{CH}_{2}$ unit cell.

ting closer to zero progressively. This phenomenon is clearly the manifestation of periodic effect on highest occupied orbitals. Hartree-Fock exhibits nonlocal orbital dependent exchange-potential, which is always deeper than the local counterpart, and thus is keeping the Fermi level at a deeper energy level. Table II also reports that with one neighbor (neig=1) the KS-based methods exhibit too wide gap and as the periodicity is building up progressively, the gap is lowered significantly.

The $X \alpha$ results differ more significantly from all the other methods, yielding higher total energies and higher Fermi levels which is due to the wrong exponential long range behavior of the LDA exchange potential. The smallest HOMOLUMO transition, the fundamental (eigenvalue) band gap is found with all the methods at the edge of the Brillouin zone $(k=1)$. As can be seen in Table III, increasing the basis set leads to a decrease of the calculated gap for all the methods, which is mainly due to the low first virtual levels when compared with HF. While the HF gap is still too high the calculated Slater's, SSP gaps are too low. $X \alpha$ provides value surprisingly close to the experimental one ${ }^{15}$ while in the literature $X \alpha$ is known for typically giving gaps which are too small. ${ }^{9}$ It is worth noting, however, that the HF gap goes through significant changes with the size of basis set as well in the $[14,24] \mathrm{eV}$ range. ${ }^{14}$ It is mainly due the downwards movement of the HF first virtual level $\epsilon_{m+1}$ while the $\epsilon_{m}$ level changes only slightly with respect to the size of the basis set. As is well known, Hartree-Fock provides too large gaps. ${ }^{14}$ Although Slater and SSP give values around the experimental one $[\sim 8.8 \mathrm{eV}$ (Refs. 15 and 31)] with the minimal basis set (Table II) the gap obtained by SSP is closer to the $X \alpha$ value. However, the band gap is narrowed significantly when the basis set is enlarged (Table III). This clearly must be attributed to the downwards movement of the first virtual levels $\epsilon_{m+1}$ and at the same time to the raising of the Fermi level $\epsilon_{m}$.

To understand the "small eigenvalue gap" problem in Figs. 1 and 2 the band structure is plotted obtained by various methods using the Clementi's minimal and double- $\zeta$ bases (Figs. 1 and 2, respectively). Analyzing and comparing systematically the band structures in Figs. 1 and 2 one can see that "new" virtual bands appear below the first virtual level obtained with the minimal basis set. The larger the basis set is, the larger the virtual space becomes which can result in the appearance of virtual states with low orbital energies. We would like to emphasize that the appearance of new low-lying virtual levels below the ones of the minimalbasis calculations is certainly not unexpected. As the basis set increases new states appear among the virtual canonical orbitals with orbital energies that form a dense subset of possible virtual energies. ${ }^{32}$ It is not easy then to separate out the virtual state which corresponds to the lowest conduction band state and also the one-electron excited states are hidden in the virtual subspace.

A more serious aspect of the small gap problem to be considered is that one has to take into account the derivative discontinuity to get the right gap when using OEP. ${ }^{1,20}$ For continuum approximations to $E_{x c}$ like LDA or GGA this discontinuity vanishes. In OEP and in our approaches, however, we have a finite derivative discontinuity. ${ }^{5,20}$ In OEP the exact band gap can be written as follows: ${ }^{20,33}$

$$
\begin{aligned}
\Delta & =\Delta_{\text {nonint }}^{\mathrm{KS}}+\Delta_{x c} \\
& =\epsilon_{\nu}^{\mathrm{KS}}(N)-\epsilon_{i}^{\mathrm{KS}}(N)+\Delta_{x c},
\end{aligned}
$$

where $N$ is the number of electrons. One has to add the $x c$ contribution ( $\Delta$ is identical with the band gap) to the difference of the Fermi ( $i$ th level) and first virtual KS one-electron 


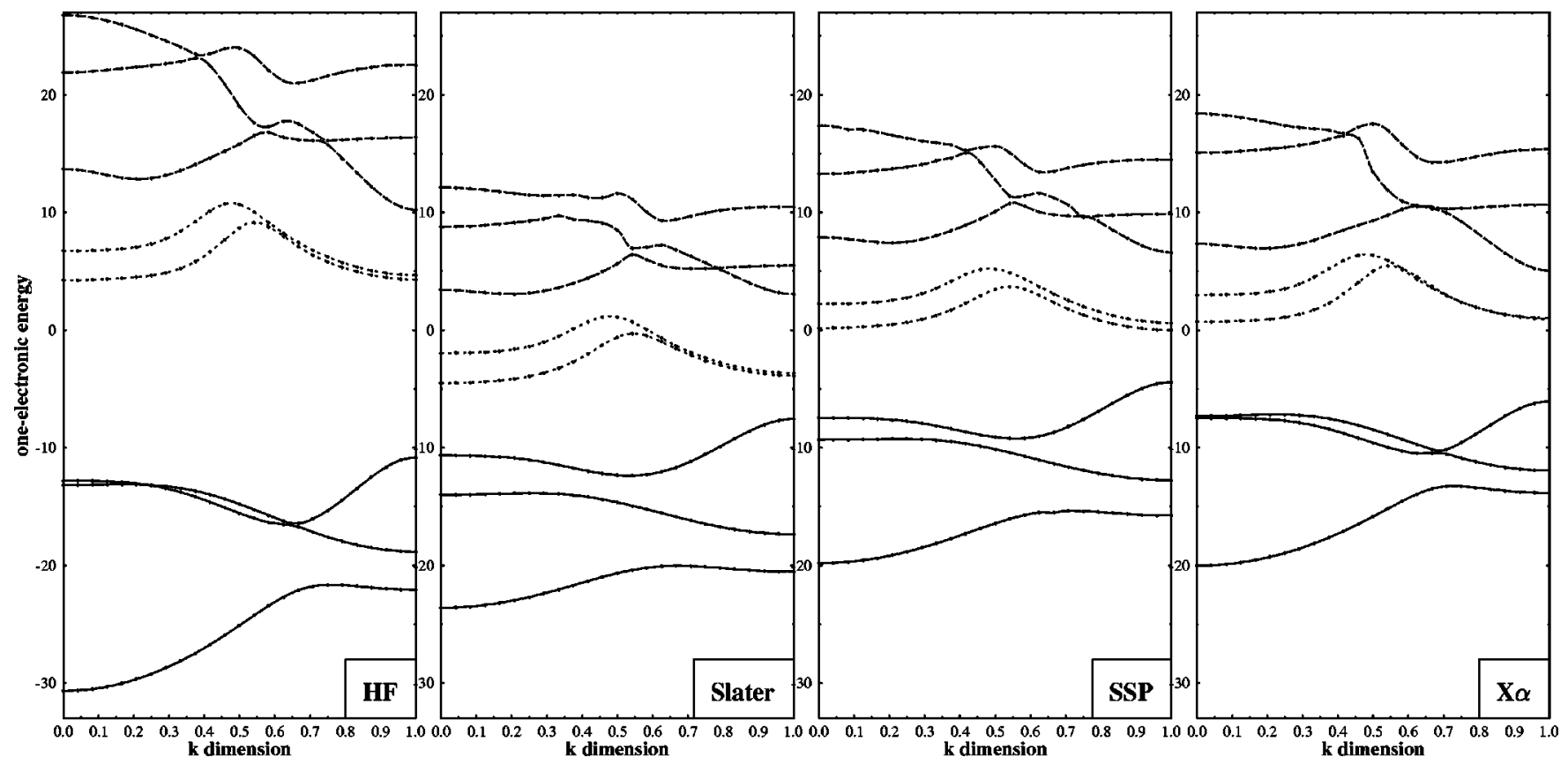

FIG. 2. The calculated valence band structure (eV) obtained by various exchange-only methods. Solid lines correspond to occupied and dashed lines to virtual levels. The two lowest dashed curves of virtual levels are of particular interest (see text). The Clementi's double- $\zeta$ basis set is used and eight neighbors are considered for the $\mathrm{CH}_{2}$ unit cell. Notations are the same as in Fig. 1.

energies (level $\nu$ ) which represent the highest valence band and the energetically lowest conduction band states, respectively. $\Delta_{\text {nonint }}^{\mathrm{KS}}$ is the Kohn-Sham eigenvalue band gap. The exchange-only contribution to the exact band gap $\Delta_{x}$ can be given $^{33}$

$$
\begin{aligned}
\Delta_{x}(i \rightarrow \nu) & =\left\langle u_{\nu}\left|\hat{\mathbf{v}}_{x}^{\mathrm{HF}}-\hat{\mathbf{v}}_{x}^{\mathrm{OEP}}[\rho]\right| u_{\nu}\right\rangle-\left\langle u_{i}\left|\hat{\mathrm{v}}_{x}^{\mathrm{HF}}-\hat{\mathrm{v}}_{x}^{\mathrm{OEP}}[\rho]\right| u_{i}\right\rangle \\
& -\langle\nu i \mid \nu i\rangle+2\langle\nu i \mid i \nu\rangle,
\end{aligned}
$$

with $\hat{\mathrm{V}}_{x}^{\mathrm{HF}}$ being the nonlocal HF exchange operator constructed from the N/2 occupied KS orbitals. This formula is coming from the first order DFT perturbation theory ${ }^{34}$ and the eigenvalue gap $\Delta_{\text {nonint }}^{\mathrm{KS}}$ represents the zeroth order term in the perturbation expansion. ${ }^{33}$ We calculated $\Delta_{x}$ using Slater and SSP methods and the results can be seen in Tables II and III. Values are also obtained when the numbers of the neighbors are minimal (neig=1). In Eq. (38) the last terms will vanish when $n$ eig $\rightarrow \infty$. Therefore terms $\langle\nu i \mid \nu i\rangle$ and $\langle\nu i \mid i \nu\rangle$ will vanish for systems with periodic boundary conditions in the limit of an infinite number of unit cells. ${ }^{33}$ We have studied the convergence of $\Delta_{x}$ and as far as the minimal basis set is concerned we found no significant change in the magnitude at neig $=5$. The discontinuity is guaranteed to be smaller than the true band gap by its definition. This is reproduced by our calculations only with small basis set. Calculations with the small basis set resulted in negative value for $\Delta_{x}$ which corrects the too large KS band gaps in the right way (Table II). With the larger basis sets $\Delta_{x}$ becomes positive and roughly represents the twice of the KS band gap. This is in accordance with the finding of Stadële et al. $^{33}$ that the corrected band gaps are close to the Hartree-Fock gap. SSP gives the value of $12.7 \mathrm{eV}$ for the true band gap which is really close to the $\mathrm{HF}$ gap $(15 \mathrm{eV})$. As Stadële et al. has pointed out, $\Delta_{x}$ equals the difference between the HF and the
OEP eigenvalue gaps, if we assume that the difference between the HF and OEP orbitals is negligible. ${ }^{33}$ Therefore, it is not surprising that such an approximation to exchangeonly OEP, like the SSP approach, provides band gap very similar to the HF one.

We got the corrected gap $6.9 \mathrm{eV}$ using the Slater's approach which is rather close to the $X \alpha$ value $6.8 \mathrm{eV}$ when the basis $(9 \mathrm{~S} / 5 \mathrm{P})$ is used. Further increase of the basis set $(11 \mathrm{~S} /$ 9P) results in even larger gap $\Delta$ for all the methods considered in this article. $X \alpha$ LDA provides a gap $7.6 \mathrm{eV}$ close to the experimental value. ${ }^{15}$ This is in accordance with the expectations since a continuum functional, like the $X \alpha$ functional, which averages over the discontinuity, should yield a gap that is reasonably close to the experimental one. The discrepancy between the calculated gaps and the experimental one must be partly attributed to the correlation contribution to the derivative discontinuity $\left(\Delta_{c}\right)$. Although we would not like to go into speculations, however, it is quite probable that $\Delta_{c}$ will provide much smaller contribution to the true band gap than $\Delta_{x}$. Further attention must be given to this in order to determine the magnitude of $\Delta_{x c}$ precisely. Nevertheless, the naked Slater exchange potential provides a corrected band gap $\Delta=9.7 \mathrm{eV}$ not far from the experiment $(\simeq 8.8 \mathrm{eV})$ when the large (11S/7P) basis set is used (Table III).

The effect of electron correlation on the gaps also should be carefully examined, however the recent publications indicate only minor importance of correlation in DFT gap calculations. ${ }^{14}$ This may well be due to the small effect of LDA correlation potential on the eigenvalues. ${ }^{9}$ Because the correlation energy is strongly dependent not only on occupied states but on the virtual states as well, no simple density functional can be expected to yield coherent gaps for different systems. Only those correlation energy functionals can provide reasonable eigenvalues and can be checked against 
experimental data by any chance, which are treated strictly at the orbital dependent level of theory.

The comparison of the Slater and SSP valence energy bands with those of $X \alpha$ and HF again shows that the shape of the bands does not change very much (Figs. 1 and 2). But the two highest valence energy bands are much further apart than in $X \alpha$ or HF. This is at least in part responsible for the small gaps we get for Slater and SSP. As already mentioned above, comparing Fig. 1 with Fig. 2 one can see that using larger basis set (Fig. 2) virtual bands are appearing below the zero energy level. This phenomenon is clearly demonstrated by all the Kohn-Sham based methods except $X \alpha$. Note that the lower three curves in both figures are the occupied valence energy levels (the deepest, core level is not plotted). The virtuals of HF do not go below zero as well. However, the first virtual levels are lowered significantly as well so that the band gap is reduced from $22 \mathrm{eV}$ to $15 \mathrm{eV}$. The highest three virtual levels in Fig. 2 are similar to the virtuals obtained by the minimal basis set (Fig. 1). The smallest energy difference from the highest occupied level to the first virtual is at the edge of the Brillouin zone $(k=1)$, however, around $k=0.5$ one can see quasidegeneracy of certain bands or even crossing of virtual levels close to the edge of the Brillouin zone (Fig. 2). Comparing Fig. 1 and Fig. 2 it is obvious that increasing the basis set shifts the valence bands upwards but does not change their shape and relative position very much. But from the same figures one can see that the shape and position of the virtual bands changed a lot when increasing the basis set. The energy bands get shifted upwards as one moves from Slater to SSP. This seems to be consistent with Gritsenko's and Krieger's work: in Gritsenko's Fig. 4 (Ref. 11) the Slater exchange potential for $\mathrm{Ne}$ and $\mathrm{Mg}$ gets shifted upwards when the response part is added. Krieger's Fig. 2 (Ref. 5) shows the same behavior for the $\mathrm{Ne}$ atom as one goes from the Slater exchange potential to better exchange potentials as KLI and exact OEP. Comparison of the Slater and SSP valence energy bands with those from $X \alpha$ and HF again show that the shape of the bands does not change very much.

The maximum of the valence band energy is appearing in the range of $[-6,-11] \mathrm{eV}$ with the lowest and highest values for $X \alpha$ and Hartree-Fock, respectively, compared to experimentally suggested values for the ionization potential of 9.6$9.8 \mathrm{eV} .{ }^{35}$ However, it must be emphasized that others give lower experimental values in the 7.6-8.8 eV range for this property. ${ }^{14,36} \mathrm{SSP}$ provides the much lower $-4.1 \mathrm{eV}$ value (Slater: $-7.6 \mathrm{eV}$ ). By density functional linear muffin-tin orbital method the value of $-5.1 \mathrm{eV}$ is obtained for helical polyethylene. ${ }^{36}$ The calculated width of the lowest valence band for $X \alpha$, HF, SSP and Slater's approach is, respectively, $6.2,9.0,4.4$, and $3.6 \mathrm{eV}$, which is to be compared with the experimental value of $7.2 \mathrm{eV} .{ }^{35}$ The total valence bandwidth is $14.0 \mathrm{eV}(X \alpha), 15.3 \mathrm{eV}$ (Slater and SSP), and $19.8 \mathrm{eV}$ (HF), compared to an experimental value of $16.2 \mathrm{eV} .{ }^{35}$ Slater and SSP perform quite well for this band width. The calculated gap between the lowest valence band and the minimum of the higher valence bands is $3.3 \mathrm{eV}(\mathrm{HF}), 1.9 \mathrm{eV}(\mathrm{X} \alpha), 3.0$ (SSP), and $3.2 \mathrm{eV}$ (Slater), respectively. These numbers have to be compared with the experimental $2.0 \mathrm{eV} .{ }^{35}$ The HF and $X \alpha$ values are in accordance with those obtained by others. ${ }^{14,15}$ The bottom of the valence band with $\sigma$ symmetry is roughly in the range of $[-31,-22] \mathrm{eV}$, which is in qualitative agreement with photoemission data ${ }^{35}$ with $2 \mathrm{eV}$ difference in average.

\section{CONCLUSIONS}

Calculations have been carried out for polyethylene with a polymer code using various orbital-dependent exchange-only potentials. The Slater potential as well as its improved version, which incorporates a step-potential for the response part of the exact exchange potential, are used and the results are compared with those obtained by Hartree-Fock or the $X \alpha$ methods. The addition of the step potential as the response part of the exact exchange to the Slater potential results in deeper total energy and somewhat wider gap for polyethylene.

To test the quality and reliability of our code, calculations have been performed in the atomic and molecular limit. The results agree with those obtained by others.

In general, we find that the band structure calculated by different $\mathbf{v}_{x}$ potentials are similar with little qualitative difference, while all of them differ significantly from HF which has valence bands significantly lower, especially in the deeper valence region and also exhibits higher virtual levels. While the band gap obtained by Clementi's minimal basis set is close to the experiment, a larger basis leads to gaps which are rather small. However, this poor performance for the gap is attached not to the approximate nature of the methods applied in this paper or not even to OEP but rather to the difficulties of gap calculations in extended systems.

Less negative Fermi levels are calculated by Kohn-Sham based orbital dependent methods than by Hartree-Fock, which comes as a surprise, because in molecules the highest occupied energy levels are very close to those of HF. On the basis of progressive improvement of the numbers of neighbors in the neighboring approach the bulk part of this difference must be attributed to periodic effects. Slater and SSP provide eigenvalue band gaps which are too narrow. In the discussion section we are speculating on the possible reasons for this unexpected phenomenon. The exchange component to the derivative discontinuity is calculated in order to correct the Kohn-Sham band gap. These calculations indicate that the discontinuity $\Delta_{x}$ is roughly twice of the Kohn-Sham eigenvalue band gap. The corrected band gaps are close to those obtained by LDA. On the basis of results reported here the approximate exchange-only potential SSP seems to provide a true band gap for polyethylene which is rather close to the Hartree-Fock gap. The simple Slater exchange potential provides a band gap in better agreement with the experiment.

\section{ACKNOWLEDGMENTS}

The authors wish to thank Dr. Ferenc Bartha for helpful discussions. This work was supported by the Flemish Science Foundation. One of us (S.K.) gratefully acknowledges financial support from the Deutsche Forschungsgemeinschaft. 
*Electronic address: sulearuca.ua.ac.be

${ }^{1}$ W. Kohn and P. Vashishta, Theory of the Inhomogeneous Electron Gas (Plenum Press, New York, 1983); R. M. Dreizler and E. K. U. Gross, Density Functional Theory (Springer, Berlin, 1990); Á. Nagy, Phys. Rep. 298, 1 (1998).

${ }^{2}$ R. T. Sharp and G. K. Horton, Phys. Rev. 90, 317 (1953).

${ }^{3}$ J. D. Talman and W. F. Shadwick, Phys. Rev. A 14, 36 (1976).

${ }^{4}$ P. Hohenberg and W. Kohn, Phys. Rev. 136, B864 (1964).

${ }^{5}$ J. B. Krieger, Y. Li, and G. J. Iafrate, Phys. Rev. A 45, 101 (1992); see also in Density Functional Theory, Vol. 337 of NATO Advanced Study Institute, Series B: Physics, edited by E. K. U. Gross and R. M. Dreizler (Plenum, New York, 1995), p. 191.

${ }^{6}$ E. Engel and S. H. Vosko, Phys. Rev. A 38, 3098 (1993); Y. Li, J. B. Krieger, and G. J. Iafrate, Chem. Phys. Lett. 191, 38 (1992); T. Grabo and E. K. U. Gross, ibid. 240, 141 (1995).

${ }^{7}$ T. Grabo and E. K. U. Gross, Int. J. Quantum Chem. 64, 95 (1997).

${ }^{8}$ T. Kotani, Phys. Rev. Lett. 74, 2989 (1995).

${ }^{9}$ D. M. Bylander and L. Kleinman, Phys. Rev. B 52, 14566 (1995); Phys. Rev. Lett. 74, 3660 (1995); 75, 4334 (1995).

${ }^{10}$ R. W. Godby, M. Schlüter, and L. J. Sham, Phys. Rev. B 36, 6497 (1987)

${ }^{11}$ O. V. Gritsenko, R. van Leeuwen, E. van Lenthe, and E. J. Baerends, Phys. Rev. A 51, 1944 (1995).

${ }^{12}$ J. P. Perdew, Electronic Structure of Solids '91 (Akademie Verlag, Berlin, 1991); J. P. Perdew, K. Burke, and M. Ernzerhof, Phys. Rev. Lett. 77, 3865 (1996); 78, 1396 ()1997.

${ }^{13}$ G. Ortiz, Phys. Rev. B 45, 11328 (1992).

${ }^{14}$ J. J. Ladik, Quantum Theory of Polymers as Solids (Plenum Press, New York, 1988).

${ }^{15}$ J. W. Mintmire, in Density Functional Methods in Chemistry, edited by J. K. Labanowski and J. W. Andzelm (SpringerVerlag, New York, 1991), p. 125; M. S. Miao, P. E. Van Camp, V. E. Van Doren, J. J. Ladik, and J. W. Mintmire, Int. J. Quantum Chem. 64, 243 (1997); Phys. Rev. B 54, 10430 (1996).

${ }^{16}$ P. Süle, O. Gritsenko, Á. Nagy, and E. J. Baerends, J. Chem. Phys. 103, 10385 (1995); P. Süle, Ph.D. thesis, Debrecen, 1996.

${ }^{17}$ J. C. Slater, Phys. Rev. 81, 385 (1951).

${ }^{18}$ R. van Leeuwen, O. V. Gritsenko, and E. J. Baerends, Analysis and Modelling of Atomic and Molecular Kohn-Sham Potential, Topics in Current Chemistry (Springer, Berlin, 1996).
${ }^{19}$ V. Sahni, J. Gruenebaum, and J. P. Perdew, Phys. Rev. B 26, 4371 (1982); J. P. Perdew and M. R. Norman, ibid. 26, 5445 (1982).

${ }^{20}$ T. Grabo, T. Kreibich, S. Kurth, and E. K. U. Gross, in The Strong Coulomb Correlations and Electronic Structure Calculations: Beyond Local Density Approximations, edited by V. Anisimov (Gordon and Breach, Amsterdam, in press).

${ }^{21}$ P. Süle (unpublished).

${ }^{22}$ J. L. Whitten, J. Chem. Phys. 39, 349 (1966).

${ }^{23}$ I. Shavitt, Methods Comput. Phys. 1963, 1.

${ }^{24}$ A. Szabó, and N. S. Ostlund, Modern Quantum Chemistry (McGraw-Hill, New York, 1989); S. Wilson, Electron Correlation in Molecules (Clarendon Press, Oxford, 1984).

${ }^{25} \mathrm{P}$. Otto, Integral and HFCO Program Package, Institute for Theoretical Chemistry, Friedrich-Alexander University, Erlangen.

${ }^{26} \mathrm{~S}$. Kurch and P. Süle, program DIOGENES, HF, LDA, and OEP polymer fortran code, Dept. Natuurkunde, TSM, RUCA, Antwerpen.

${ }^{27}$ S. Hirata and S. Iwata, J. Chem. Phys. 108, 7901 (1998).

${ }^{28}$ G. C. Lie and E. Clementi, J. Chem. Phys. 60, 1275 (1974).

${ }^{29}$ Gaussian 94, Revision E.2, M. J. Frisch, G. W. Trucks, H. B. Schlegel, P. M. W. Gill, B. G. Johnson, M. A. Robb, J. R. Cheeseman, T. Keith, G. A. Petersson, J. A. Montgomery, K. Raghavachari, M. A. Al-Laham, V. G. Zakrzewski, J. V. Ortiz, J. B. Foresman, J. Cioslowski, B. B. Stefanov, A. Nanayakkara, M. Challacombe, C. Y. Peng, P. Y. Ayala, W. Chen, M. W. Wong, J. L. Andres, E. S. Replogle, R. Gomperts, R. L. Martin, D. J. Fox, J. S. Binkley, D. J. Defrees, J. Baker, J. P. Stewart, M. Head-Gordon, C. Gonzalez, and J. A. Pople, Gaussian, Inc., Pittsburgh, Pa, 1995.

${ }^{30}$ M. Levy and J. P. Perdew, Phys. Rev. A 32, 2010 (1985).

${ }^{31}$ K. J. Less andd E. G. Wilson, J. Phys. C 6, 3110 (1973).

${ }^{32} \mathrm{~F}$. Bartha (private communication).

${ }^{33}$ M. Städele, J. A. Majewski, P. Vogl, and A. Görling, Phys. Rev. Lett. 79, 2089 (1997).

${ }^{34}$ A. Görling, Phys. Rev. A 54, 3912 (1996).

${ }^{35}$ K. Seki, N. Ueno, U. O. Karlsson, R. Engelhardt, and E.-E. Koch, Chem. Phys. 105, 247 (1986).

${ }^{36}$ M. Springborg and M. Lev, Phys. Rev. B 40, 3333 (1989).

${ }^{37}$ Y. Li, J. B. Krieger, and G. J. Iafrate, Phys. Rev. A 46, 5453 (1992). 